ITC $1 / 47$

Journal of Information Technology and Control

Vol. 47 / No. 1/ 2018

pp. $140-150$

DOI 10.5755/j01.itc.47.1.19982

(C) Kaunas University of Technology

\section{H $\propto$ Control of Network Control System for Singular Plant}

Received 2018/01/19

Accepted after revision 2018/02/05

\title{
$\mathrm{H} \infty$ Control of Network Control System for Singular Plant
}

\section{Wei Wei}

School of Computer Science and Engineering, Xi'an University of Technology, Xi’an 710048, China; Qilu University of Technology (Shandong Academy of Sciences); Shandong provincial Key Laboratory of Computer Network; e-mail:weiwei@xaut.edu.cn

\section{Xunli Fan}

School of Information Science \& Technology, Northwest University, Xi'an 710127, China; e-mail:xunlfan@nwu.edu.cn

\section{Marcin Woźniak}

Institute of Mathematics, Silesian University of Technology, Kaszubska 23, 44-100 Gliwice, Poland; e-mail: Marcin.Wozniak@polsl.pl

\section{Houbing Song}

Department of Electrical, Computer, Software, and Systems Engineering, Embry-Riddle Aeronautical University, Daytona Beach, FL 32114-3900, USA; e-mail: h.song@ieee.org

\section{Wei-Li}

The Center for Distributed and High Performance Computing, School of Information Technologies, The University of Sydney, Sydney, Australia; e-mail: liwei@cs.usyd.edu.au

\section{Ye Li}

School of Computer Science and Engineering, Xi'an University of Technology, Xi’an 710048, China; Qilu University of Technology (Shandong Academy of Sciences); Shandong provincial Key Laboratory of Computer Network

\section{Peiyi Shen}

National school of Software, Xidian University, Xi’an 710071, P.R.China, China; e-mail: pyshen@xidian.edu.cn 
This paper investigates $H_{\infty}$ control method for a class of Singular Network Control Systems (SNCS) based on singular plant. Considering the network delay, external disturbance, impulse behavior and structural instability of singular plant, the $H_{\infty}$ control of SNCS with state feedback and dynamic output feedback are investigated respectively by approach of Linear Matrix Inequality (LMI). The existence of the $H_{\infty}$ control law, the solving of the $H_{\infty}$ control law and the disturbance degree are discussed in the following sections of the paper. Simulation results illustrate the effectiveness and feasibility of the given approach.

KEYWORDS: Singular Network Controls, $H_{\infty}$ control, Linear Matrix Inequality, Network delay.

\section{Introduction}

Network Control System (NCS) is a distributed and a real-time feedback control system where the system node situated at different geographical position exchanges state information and control information with the controller through a communication network [20]. Network bandwidth and restraint of communication mechanism such as network delay and data packet loss exist typically in network communication channel, which makes NCS loses invariability, integrality, causality and certainty [18], and due to this fact the study of NCS is more complicated and challenging.

The traditional control theories and methods are not suitable for NCS, which makes rapid development over the past few years. Since the end of the last century, the research of NCS experiences the process from simple to complex, from single to comprehensive and from special to general. A large number of results have been reported, for instance, system complexity analysis [23], quantized dynamic output feedback control [3], observer-based controller design [25], state estimation and stabilization [6], $H_{\infty}$ control method [24], fault-tolerant control [4], guaranteed cost control [8], co-design [10].

The results in the existing literature are focused on linear system. However, the study of SNCS based on singular system has not been addressed intensively. The dynamics of singular system is quite different from normal linear system and have many characteristics such as no causality, no solution, no uniqueness and structure instability, etc. [22]. In fact, the research on SNCS is still in the primary stage, and the existing results are limited to system modeling, stability analysis and control method [1-2, 5, 11-12, 14-17].

This paper aims to study the stabilization and $H_{\infty}$ control method for a class of SNCS subject to the double characteristics of a singular systems and NCS. Net- work delay, input disturbance of limited energy, and impulse behavior are taken into consideration. The $H_{\infty}$ control method of SNCS with state feedback and dynamic output feedback are presented respectively by means of LMI. The existence $H_{\infty}$ control law, $H_{\infty}$ control law approach and disturbance attenuation degree in different feedback are presented. Finally, a simulation is given to illustrate the effectiveness of the proposed method [7, 9, 13, 19,21].

\section{Schur Formula and Schur Complement Lemma}

Let $A \in R^{r \times r}, B \in R^{r \times(n-r)}, C \in R^{(n-r) \times r}, D \in R^{(n-r) \times(n-r)}$, and $A$ be an invertible matrix. The Schur formula has the following three forms:

(i)

$$
\begin{aligned}
& {\left[\begin{array}{cc}
A & B \\
0 & \mathrm{D}-\mathrm{CA}^{-1} \mathrm{~B}
\end{array}\right]=\left[\begin{array}{cc}
I_{(r)} & 0 \\
-C A^{-1} & I_{(n-r)}
\end{array}\right]\left[\begin{array}{ll}
A & B \\
C & D
\end{array}\right],} \\
& \text { (ii) }\left[\begin{array}{cc}
A & 0 \\
C & D-C A^{-1} B
\end{array}\right]=\left[\begin{array}{cc}
A & B \\
C & D
\end{array}\right]\left[\begin{array}{cc}
I_{(r)} & -A^{-1} B \\
0 & I_{(n-r)}
\end{array}\right] \text {, }
\end{aligned}
$$

(iii) $\left[\begin{array}{cc}A & 0 \\ 0 & D-C A^{-1} B\end{array}\right]=$

$$
=\left[\begin{array}{cc}
I_{(r)} & 0 \\
-C A^{-1} & I_{(n-r)}
\end{array}\right]\left[\begin{array}{cc}
A & B \\
C & D
\end{array}\right]\left[\begin{array}{cc}
I_{(r)} & -A^{-1} B \\
0 & I_{(n-r)}
\end{array}\right],
$$

in which, e.g., $I_{(r)}$ denotes identity matrix of the size $r \times r$. The other identity matrices are of sizes that fit the Schur lemma.

\section{Schur Complement Lemma:}

Let $\mathrm{Q} \in R^{r \times r}, \mathrm{~S} \in R^{r \times(n-r)}, \mathrm{R} \in R^{(n-r) \times(n-r)}$ and $\left[\begin{array}{cc}Q & S \\ S^{T} & R\end{array}\right]<0$. Then if and only if

(i) $\mathrm{R}<0, \mathrm{Q}-S R^{-1} S^{T}<0$

or

(ii) $\mathrm{Q}<0, \mathrm{R}-S^{T} \mathrm{Q}^{-1} \mathrm{~S}<0$. 
Proof: Since the contract transform does not change the matrix positive definition, we first prove that $\left[\begin{array}{cc}Q & S \\ S^{T} & R\end{array}\right]<0$ is equivalent to $\mathrm{R}<0, \mathrm{Q}-S R^{-1} S^{T}$.

If $Q<0$, through the Schur formula, we have the following:

$$
\begin{aligned}
& {\left[\begin{array}{cc}
I_{(r)} & -S R^{-1} \\
0 & I_{(n-r)}
\end{array}\right]\left[\begin{array}{cc}
Q & S \\
S^{T} & R
\end{array}\right]\left[\begin{array}{cc}
I_{(r)} & 0 \\
-R^{-1} S^{T} & I_{(n-r)}
\end{array}\right]=} \\
& =\left[\begin{array}{cc}
Q-S R^{-1} S^{T} & 0 \\
0 & R
\end{array}\right] .
\end{aligned}
$$
Correspondingly, $\left[\begin{array}{cc}Q & S \\ S^{T} & R\end{array}\right]<0$ is equivalent to $\mathrm{R}<0$,
$\mathrm{Q}-S R^{-1} S^{T}<0$.

\section{[End of Proof]}

Notes: Q- $S R^{-1} S^{T}$ is the Schur complement of Q.

\section{Problem Description}

The singular sample of the network control system is described as presented in [1].

In Figure 1, $u, w$ and $z$ are control input, measurement state or measurement output, input external disturbance and expectation output, and $\tau$ is network-induced delay. The aim of positioning is to guarantee stable running of the system independently of any external disturbances so that the expected output of the system is not affected.

\section{Figure 1}

General Structure of SNCS

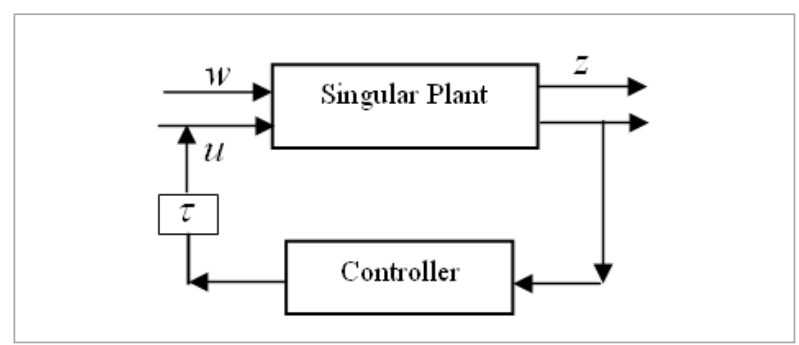

For singular plant, its state response contains not only the exponential term as normal systems, but also the pulse term and input derivative item, which will make the whole system has a pulse behavior. The pulse behavior decreases not only the system performance and even leads to the unstable state, which is a fatal destructiveness to the system. For network communication, due to the limited network bandwidth and the restraint of communication mechanism, the network communication obtains uncertainty and complexity.
The presented model shows a singular system state:

$$
\left\{\begin{array}{l}
E_{x} \&(t)=A x(t)+B u\left(t-l+H_{0} w(t)\right) \\
y(t)=C_{1} X(t)+H_{1} w(t) \\
z(t)=C_{2} x(t)+H_{2} w(t)
\end{array}\right\},
$$

where $\mathrm{x}(\mathrm{t}) \in R^{n} \mathrm{u}(\mathrm{t}) \in R^{m,} \mathrm{y}(\mathrm{t}) \in R^{l}$ and $\mathrm{z}(\mathrm{t}) \in R^{l}$ are state vector, control input'vector, output vector and expectation output vector, respectively. A $\in R^{n \times n}$, B $\in R^{n_{\times} m}$ and $C_{1}, C_{2} \in R^{n_{\times} l}$ are constant matrices, $\mathrm{E} \in$ $R^{n \times n}$ is a singular matrix; $\mathrm{w}(\mathrm{t})$ is finite energy external disturbance, and $H_{0}, H_{1}, H_{2}$ are constant matrices.

When the singular plant is regular and impulse free, the equation (1) can be equivalently transformed as:

$$
\left\{\begin{array}{l}
x_{1} \&(t)=A_{1} x_{1}(t)+B_{1} u(t-l)+W_{1} w(t) \\
x_{2} \&(t)=x_{2}(t)+B_{2}(t-1)+W_{2} w(t) \\
y(t)=C_{11} x_{1}(t)+C_{12} x_{2}(t)+H_{1} w(t) \\
z(t)=C_{21} x_{1}(t)+C_{22} x_{2}(t)+H_{2} w(t)
\end{array} .\right.
$$

The state feedback control is

$u(k)=\left[\begin{array}{ll}K_{1} & K_{2}\end{array}\right]\left[\begin{array}{l}x_{1}(k) \\ x_{2}(k)\end{array}\right]$.

Let $\hat{x}=\left[\mathrm{x}_{1}^{T}(k) u^{T}(k-1)\right]^{\mathrm{T}}$, the state feedback SNCS close-loop model is

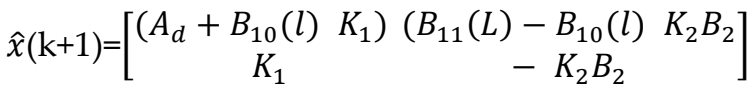

$$
\begin{aligned}
& \hat{x}+\left[\begin{array}{ccc}
W_{0}-B_{10}(l) & K_{2} & W_{2} \\
- & K_{2} W
\end{array}\right] \mathrm{w}(\mathrm{k}) .
\end{aligned}
$$

The dynamic output feedback controller is

$$
\left\{\begin{array}{c}
x_{c}(k+1)=A_{c} x_{c}(k)+B_{c} y(k) \\
u(k)=C_{c} x_{c}(k)
\end{array} .\right.
$$

Let $\bar{x}=\left[\begin{array}{lll}\mathrm{x}_{1}^{T} & \mathrm{x}_{c}^{T} & u^{T}\end{array}\right]^{\mathrm{T}}$, the SNCS close-loop model is as follows:

$$
\begin{gathered}
\bar{x}(k+1)=\left[\begin{array}{ccc}
A_{d} & B_{10}(l) C_{c} & b_{11}(l) \\
B_{c} & A_{c} & -B_{c} C_{12} B_{2} \\
0 & C_{c} & 0
\end{array}\right] \\
\bar{x}(k)+\left[\begin{array}{c}
W_{0} \\
B_{c} H_{1}-B c C_{12} W_{2} \\
0
\end{array}\right] w(k) .
\end{gathered}
$$


Whether the system uses state feedback and output feedback or not, the SNCS close-loop system model is a linear normal system depending on time delay $\tau$.

\section{$\boldsymbol{H}_{\infty}$ Control}

\section{State feedback $\boldsymbol{H}_{\infty}$ control}

Theorem 1: If there exist positive definite matrices $\bar{S}$, $\bar{R}$ such that

$$
\left[\begin{array}{cccc}
-\bar{S} & 0 & \bar{S} M_{1}^{T} & \bar{S} K_{1}^{T} \\
0 & -\bar{R} & \bar{R} M_{2}^{T} & \bar{R} M_{3}^{T} \\
M_{1} \bar{S} & M_{2} \bar{R} & -\bar{S} & 0 \\
K_{1} \bar{S} & M_{3} \bar{R} & 0 & -\bar{R}
\end{array}\right]<0
$$

where $M_{1}=A_{d}+B_{10}(l) K_{1}, M_{2}=B_{11}(l)-B_{10}(l) K_{2} B_{2}$ and $M_{3}=-K_{2} B_{2}$, then the system (2) is asymptotically stable.

Proof: Choose positive definite matrices S and R and define a Lyapunov function as follows:

$\mathrm{V}(\mathrm{k})=\mathrm{x}_{1}^{T}(k) S x_{1}(k)+u^{T}(k-1) \mathrm{Ru}(\mathrm{k}-1)$.

Then the forward differential of $\mathrm{V}(\mathrm{k})$ is $\nabla \mathrm{V}(\mathrm{k})=\hat{x}(k)^{\mathrm{T}} \prod \hat{x}(k)$, where

$\Pi=\left[\begin{array}{cc}M_{1}^{T} S M_{1}+K_{1}^{T} R K_{1}-S & M_{1}^{T} S M_{2}+K_{1}^{T} R K_{3} \\ M_{2}^{T} S M_{1}+M_{3}^{T} R K_{1} & M_{2}^{T} S M_{2}+M_{3}^{T} R M_{3}-R\end{array}\right]$, $M_{1}=A_{d}+B_{10} K_{1}, M_{2}=B_{11}-B_{10} K_{2} B_{2}, M_{3}=-K_{2} B_{2}$,

$\hat{x}=\left[\begin{array}{cc}x_{1}^{T}(k) & u^{T}(k-1)\end{array}\right]^{\mathrm{T}}$.

By Lyapunov stability theory, if $\Delta \mathrm{V}(\mathrm{k})<0$, then the system (2) is asymptotically stable and asymptotical stability condition is

$$
\left[\begin{array}{cc}
M_{1}^{T} S M_{1}+K_{1}^{T} R K_{1}-S & M_{1}^{T} S M_{2}+K_{1}^{T} R K_{3} \\
M_{2}^{T} S M_{1}+M_{3}^{T} R K_{1} & M_{2}^{T} S M_{2}+M_{3}^{T} R M_{3}-R
\end{array}\right]<0 .
$$

By Schur complement lemma, the equation (3) can be transformed to

$$
\left[\begin{array}{cccc}
-S & 0 & M_{1}^{T} & K_{1}^{T} \\
0 & -R & M_{2}^{T} & M_{3}^{T} \\
M_{1} & M_{2} & -S^{-1} & 0 \\
K_{1} & M_{3} & 0 & -R^{-1}
\end{array}\right] .
$$

Multiplying diag ( $S^{-1}, R^{-1}, I, I$ ) on the left-hand side and the right-hand side of the equation it is derived that

$$
\left[\begin{array}{cccc}
-S^{-1} & 0 & S^{-1} M_{1}^{T} & S^{-1} K_{1}^{T} \\
0 & -R^{-1} & R^{-1} M_{2}^{T} & R^{-1} M_{3}^{T} \\
M_{1} S^{-1} & M_{2} R^{-1} & -S^{-1} & 0 \\
K_{1} S^{-1} & M_{3} R^{-1} & 0 & -R^{-1}
\end{array}\right],
$$

where we denote $\bar{S}=S^{-1}, \bar{R}=R^{-1}$, then the equation is equivalent to (3).

[End of Proof]

Theorem 2: For the singular plant (1), under state feedback controller, for given $\gamma>0$, if there exists symmetric positive definite matrices $S, R$, such that

$$
\left[\begin{array}{cccccc}
-S & 0 & 0 & M_{1}^{T} & K_{1}^{T} & C_{21}^{T} \\
0 & -R & 0 & M_{2}^{T} & M_{3}^{T} & M_{4}^{T} \\
0 & 0 & -\gamma^{2} I & W_{2}^{T} & W_{3}^{T} & W_{4}^{T} \\
M_{1} & M_{2} & W_{3} & 0 & 0 & 0 \\
M_{1} & M_{3} & W_{4} & 0 & -R^{-1} & 0 \\
C_{21} & M_{4} & W_{5} & 0 & 0 & -1
\end{array}\right]<0
$$

where $M_{1}=A_{d}+B_{10} K_{1}, M_{2}=B_{11}-B_{10} K_{2} B_{2}, M_{3}=-K_{2} B_{2}$, $M_{4}=-C_{22} B_{2}, W_{5}=H_{2}-C_{22} W_{2}, W_{3}=W_{0}-B_{10}(l) K_{2} B_{2}$, $W_{4}=-K_{2} W_{2}$, then the singular plant model (1) will realize second best state feedback $\boldsymbol{H}_{\infty}$ control.

Proof: The external disturbance is taken into account, in order to make the following equation exist $\|\mathrm{z}(\mathrm{k})\|_{2} \leq \gamma\|\mathrm{w}(\mathrm{k})\|_{2}$. Let $J_{Z}=\sum_{k=0}^{\infty}\left[z^{T}(k) z(k)-\right.$ $\left.\gamma^{2} w^{T}(k) w(k)\right]$, we can take positive definite matrices $\mathrm{S}, \mathrm{R}$, and define a Lyapunov function $\mathrm{V}(\mathrm{k})$ as follows: $\mathrm{V}(\mathrm{k})=\mathrm{x}_{1}^{T}(k) S x_{1}(k)+u^{T}(k-1) R u(k-1)$.

For the system (2), when it satisfies Theorem 1 , it is asymptotically stable in the zero initial conditions $\forall \mathrm{w}(\mathrm{k}) \in L_{2}[0, \infty)$ it is derived that $\sum_{k=0}^{\infty}\left[z^{T}(k) z(k)-\gamma^{2} w^{T}(k) w(k)+\Delta V(k)\right]<0$.

Let us denote $M_{4}=-C_{22} B_{2}, W_{3}=W_{0}-B_{10}(l) K_{2} B_{2}$, $W_{4}=-K_{2} W_{2}, W_{5}=H_{2}-C_{22} W_{2}$, so that it is derived that $x^{T} \Phi \mathrm{x}<0$, where

$$
\begin{aligned}
& \mathrm{x}=\left[\begin{array}{ll}
\mathrm{x}_{1}^{T}(k) & u^{T}(k-1)
\end{array}\right. \\
& \left.w^{T}(k)\right]^{\mathrm{T}}, \Phi=\left[\begin{array}{ccc}
A_{11} & * & * \\
A_{21} & A_{22} & * \\
A_{31} & A_{32} & A_{33}
\end{array}\right]<0 .
\end{aligned}
$$

$$
A_{11}=M_{1}^{T} S M_{1}+K_{1}^{T} R K_{1}-S+C_{21}^{T} C_{21} \text {, }
$$


$A_{21}=M_{2}^{T} S M_{1}+M_{3}^{T} R K_{1}+M_{4}^{T} C_{21}$,

$A_{22}=M_{2}^{T} S M_{1}+M_{3}^{T} R M_{3}-R+M_{4}^{T} M_{4}$,

$A_{31}=W_{3}^{T} S M_{1}+W_{4}^{T} R+W_{5}^{T} C_{21}$,

$A_{32}=W_{3}^{T} S M_{2}+W_{4}^{T} R+W_{5}^{T} M_{4}$,

$A_{33}=W_{3}^{T} S W_{3}+W_{5}^{T} W_{5}+W_{4}^{T} R M_{4}-\gamma^{2} I$.

By Schur complement, Equation (4) can be transformed as

$$
\left[\begin{array}{ccccc}
-S+C_{21}^{T} C_{21} & C_{21}^{T} M_{4} & C_{21}^{T} W_{5} & M_{1}^{T} & K_{1}^{T} \\
C_{21}^{T} C_{21} & -R+M_{4}^{T} M_{4} & M_{4}^{T} W_{5} & M_{2}^{T} & M_{3}^{T} \\
W_{5}^{T} C_{21} & W_{5}^{T} M_{4} & W_{5}^{T} W_{5}-\gamma^{2} I & W_{3}^{T} & W_{4}^{T} \\
M_{1} & M_{2} & M_{3} & -S^{-1} & 0 \\
K_{1} & M_{3} & W_{4} & 0 & -R^{-1}
\end{array}\right]<0 .
$$

Similarly, by further transforming, we can derive (4). [End of Proof]

Theorem 3: For the singular plant (1), under the action of state feedback controller, if there exist symmetric positive definite matrices $\widehat{S}, \hat{R}$, matrices $Y_{1}, Y_{2}$, $Y_{3}$, scalars $\varepsilon>0, \varepsilon_{1}>0, \beta>0$ and compatible dimension unit matrix $I$, such that

$\left[\begin{array}{cccccccccc}-\hat{S} & * & * & * & * & * & * & * & * & * \\ 0 & -\hat{R} & * & * & * & * & * & * & * & * \\ 0 & 0 & -\beta I & * & * & * & * & * & * & * \\ A_{\mathrm{d}} \hat{S}+B_{10} Y_{1} & B_{11} \hat{R} & W_{0} & -\hat{S} & * & * & * & * & * & * \\ Y_{1} & 0 & 0 & 0 & -\hat{R} & * & * & * & * & * \\ C_{21} \hat{S} & -C_{22} B_{2} \hat{R} & H_{2}-C_{22} W_{2} & 0 & 0 & -I & * & * & * & * \\ 0 & B_{2} \hat{R} & W_{2} & 0 & 0 & 0 & -\varepsilon I & * & * & * \\ 0 & B_{2} \hat{R} & W_{2} & 0 & 0 & 0 & 0 & -\varepsilon_{1} I & * & * \\ 0 & 0 & 0 & Y_{3} B_{10}{ }^{\mathrm{T}} & 0 & 0 & 0 & 0 & -\varepsilon I & * \\ 0 & 0 & 0 & 0 & Y_{2} & 0 & 0 & 0 & 0 & -\varepsilon_{1} I\end{array}\right]<0$.

(6)

The $\boldsymbol{H}_{\infty}$ control law is

$\mathrm{u}(\mathrm{k})=\left[Y_{1} \hat{S}^{-1} Y_{2}^{T} / \varepsilon_{1}\right]\left[\begin{array}{l}x 1(k) \\ x 2(k)\end{array}\right]$.

Proof: For plant (1), if second best state feedback c $\boldsymbol{H}_{\infty}$ control law exists, then Theorem 2 is established. Spread out $M_{1} \sim M_{4}, W_{3} \sim W_{5}$, and then Equation (4) in Theorem 2 can be expressed as
$\left[\begin{array}{cccccc}-S & * & * & * & * & * \\ 0 & -R & 0 & * & * & * \\ 0 & 0 & -\gamma^{2} I & * & * & * \\ A_{d}+B_{10} K_{1} & B_{11} & W_{0} & -S^{-1} & * & * \\ C & -K_{2} B_{2} & -K_{2} W_{2} & 0 & -R^{-1} & * \\ C_{21} & -C_{22} B_{2} & H_{2}-C_{22} W_{2} & 0 & 0 & -1\end{array}\right]<0$.

(8)

Equation (8) can be rewritten as:

$\left[\begin{array}{cccccc}-S & * & * & * & * & * \\ 0 & -R & 0 & * & * & * \\ 0 & 0 & -\gamma^{2} I & * & * & * \\ A_{d}+B_{10} K_{1} & B_{11} & W_{0} & -S^{-1} & * & * \\ C & -K_{2} B_{2} & -K_{2} W_{2} & 0 & -R^{-1} & * \\ C_{21} & -C_{22} B_{2} & H_{2}-C_{22} W_{2} & 0 & 0 & -1\end{array}\right]+$

$+\left[\begin{array}{l}0 \\ 0 \\ 0 \\ -B_{10} K_{2} \\ 0 \\ 0\end{array}\right] I\left[\begin{array}{llllll}0 & B_{2} & W_{2} & 0 & 0 & 0\end{array}\right] I^{T}\left[\begin{array}{l}0 \\ 0 \\ 0 \\ -B_{10} K_{2} \\ 0 \\ 0\end{array}\right]^{T}<0$

(9)

From Schur Lemma 1, we can say that the above (9) exists, if and only if there is a scalar $\varepsilon>0$, such that

$\left[\begin{array}{cccccc}-S & * & * & * & * & * \\ 0 & -R & 0 & * & * & * \\ 0 & 0 & -\gamma^{2} I & * & * & * \\ A_{d}+B_{10} K_{1} & B_{11} & W_{0} & -S^{-1} & * & * \\ C & -K_{2} B_{2} & -K_{2} W_{2} & 0 & -R^{-1} & * \\ C_{21} & -C_{22} B_{2} & H_{2}-C_{22} W_{2} & 0 & 0 & -1\end{array}\right]+$

$+\varepsilon\left[\begin{array}{l}0 \\ 0 \\ 0 \\ -B_{10} K_{2} \\ 0 \\ 0\end{array}\right]^{T}\left[\begin{array}{l}0 \\ 0 \\ 0 \\ -B_{10} K_{2} \\ 0 \\ 0\end{array}\right]^{T}+$

${ }^{+} \varepsilon_{-}{ }^{1}\left[\begin{array}{llllll}0 & B_{2} & W_{2} & 0 & 0 & 0\end{array}\right]^{T}\left[\begin{array}{llllll}0 & B_{2} & W_{2} & 0 & 0 & 0\end{array}\right]<0$ 
By Schur complement, Equation (10) can be transformed as

\begin{tabular}{|c|c|c|c|c|c|c|}
\hline$-S$ & $*$ & $*$ & $*$ & $*$ & $*$ & 0 \\
\hline 0 & $-R$ & $*$ & $*$ & $*$ & $*$ & $B_{2}^{\mathrm{T}}$ \\
\hline 0 & 0 & $-\gamma^{2} I$ & $*$ & $*$ & $*$ & $W_{2}^{\mathrm{T}}$ \\
\hline$A_{\mathrm{d}}+B_{10} K_{1}$ & $B_{11}$ & $W_{0}$ & $-S^{-1}+\varepsilon B_{10} K_{2}\left(B_{10} K_{2}\right)^{\mathrm{T}}$ & $*$ & $*$ & 0 \\
\hline$K_{1}$ & $-K_{2} B_{2}$ & $-K_{2} W_{2}$ & 0 & $-R^{-1}$ & $*$ & 0 \\
\hline$C_{21}$ & $-C_{22} B_{2}$ & $H_{2}-C_{22} W_{2}$ & 0 & 0 & $-I$ & 0 \\
\hline 0 & $B_{2}$ & $W_{2}$ & 0 & 0 & 0 & $-\varepsilon I\rfloor$ \\
\hline
\end{tabular}

Similarly, it is derived that

$\left[\begin{array}{cccccccc}-S & * & * & * & * & * & * & 0 \\ 0 & -R & * & * & * & * & * & B_{2}^{\mathrm{T}} \\ 0 & 0 & -\gamma^{2} I & * & * & * & * & W_{2}^{\mathrm{T}} \\ A_{\mathrm{d}}+B_{10} K_{1} & B_{11} & W_{0} & -S^{-1}+\varepsilon B_{10} K_{2}\left(B_{10} K_{2}\right)^{\mathrm{T}} & * & * & * & 0 \\ K_{1} & 0 & 0 & 0 & -R_{1}^{-1}+\varepsilon_{2} K_{2} K^{\mathrm{T}} & * & * & 0 \\ C_{21} & -C_{22} B_{2} & H_{2}-C_{22} W_{2} & 0 & 0 & -I & * & 0 \\ 0 & B_{2} & W_{2} & 0 & 0 & 0 & -\varepsilon I & 0 \\ 0 & B_{2} & W_{2} & 0 & 0 & 0 & 0 & -\varepsilon_{1} I\end{array}\right]<0$.

State feedback $\boldsymbol{H}_{\infty}$ controller parameter is $K_{1}=Y_{1} \hat{S}^{-1}, K_{2}=Y_{2}^{T} / \varepsilon_{1=} Y_{3}^{T} / \varepsilon$.

The state feedback $\boldsymbol{H}_{\infty}$ control law (7) is obtained.

[End of Proof]

\section{Dynamic output feedback $H_{\infty}$ control}

Theorem 4 when the external disturbance is not taken into account, under the action of dynamic output feedback controller, if there exist positive $\tilde{P}, \widetilde{Q}, \tilde{S}$, such that

$$
\left[\begin{array}{cccccc}
-\widetilde{P} & * & * & * & * & * \\
0 & -\widetilde{Q} & 0 & * & * & * \\
0 & 0 & -\widetilde{S} & * & * & * \\
A_{d} & M_{5} \widetilde{Q} & M_{6} \widetilde{S} & -\widetilde{P} & * & * \\
M_{2} \widetilde{P} & A_{c} \widetilde{Q} & M_{8} \widetilde{S} & 0 & -\widetilde{Q} & * \\
0 & C_{c} \widetilde{Q} & 0 & 0 & 0 & -\widetilde{S}
\end{array}\right],
$$

where $\quad M_{5}=B_{10}(L) C_{C}, \quad M_{6}=B_{11}(L), \quad M_{7}=B_{c} C_{11}$, $M_{8}=-B_{c} C_{12} B_{2}$, then the system (2) is asymptotically stable.

Proof: Denote $M_{5}=B_{10}(L) C_{C}, M_{6}=B_{11}(L), M_{7}=B_{c} C_{11}$, $M_{8}=-B_{c} C_{12} B_{2}, W_{6}=B_{c}\left(H_{1}-C_{12} W_{2}\right)$, so that Equation (2) can be written as

$$
\bar{x}(k+1)=\left[\begin{array}{ccc}
A_{d} & M_{5} & M_{6} \\
M_{7} & A_{c} & M_{8} \\
0 & M_{5} & 0
\end{array}\right] \bar{x}(k)+\left[\begin{array}{c}
W_{0} \\
W_{6} \\
0
\end{array}\right] w(k) .
$$

When the external disturbance of the system is not taken into account, choose positive definite matrices $P, Q, S$ and define a Lyapunov function as follows:

$\mathrm{V}(\mathrm{k})=\mathrm{x}_{1}^{T}(k) P(k)+x_{c}{ }^{T}(k) \mathrm{Q} x_{c}(\mathrm{k})+. u^{T}(k-1) \mathrm{Su}(\mathrm{k}-1)$

Then the forward differential of $V(k)$ along trajectory of close-loop system (6) is as follows: 


$$
\begin{aligned}
& \Delta \mathrm{V}(\mathrm{k})= \\
& \left(\mathrm{x}_{1}^{T}(k) A_{d}{ }^{T}+\mathrm{x}_{1}^{T}(k) M_{5}{ }^{T}+u^{T}(k-1) M_{6}{ }^{T} P A_{d} x_{1}(k)+\right. \\
& \left(\mathrm{x}_{1}^{T}(k) A_{d}{ }^{T}+x_{c}{ }^{T}(k) M_{5}{ }^{T}+u^{T}(k-1) M_{6}{ }^{T} P M_{5} x_{c}(\mathrm{k})+\right. \\
& \left(\mathrm{x}_{1}^{T}(k) A_{d}{ }^{T}+x_{c}{ }^{T}(k) M_{5}{ }^{T}+u^{T}(k-1) M_{6}{ }^{T} P M_{6} u(k-1)+\right. \\
& \left(\mathrm{x}_{1}^{T}(k) M_{7}{ }^{T}+x_{c}{ }^{T}(k) A_{c}{ }^{T}+u^{T}(k-1) M_{8}{ }^{T}\right) Q M_{7} x_{1}(k)+ \\
& \left(\mathrm{x}_{1}^{T}(k) M_{7}{ }^{T}+x_{c}{ }^{T}(k) A_{c}{ }^{T}+u^{T}(k-1) M_{8}{ }^{T}\right) Q A_{C} x_{C}(k)+ \\
& \left(\mathrm{x}_{1}^{T}(k) M_{7}{ }^{T}+x_{c}{ }^{T}(k) A_{c}{ }^{T}+u^{T}(k-1) M_{8}{ }^{T}\right) Q M_{8} u(k-1)+ \\
& x_{c}{ }^{T}(k) C_{C}{ }^{T} S C_{C} x_{C}(k)-x_{c}{ }^{T}(k) Q x_{C}(k)- \\
& u^{T}(k-1) S u(k-1) .
\end{aligned}
$$

We define $\tilde{x}(k)=\left[\left(\mathrm{x}_{1}^{T}(k) x_{c}{ }^{T}(k) u^{T}(k-1)\right]^{\mathrm{T}}\right.$, for which the above equation can be written as $\nabla V(k)=\tilde{x}^{\mathrm{T}} \Psi \tilde{x}$,

$\varphi=\left[\begin{array}{ccc}D_{11} & * & * \\ D_{21} & D_{22} & * \\ D_{31} & D_{32} & D_{33}\end{array}\right]$,

where $D_{11}=A_{d}{ }^{T} P A_{d}+M_{7}{ }^{T} Q M_{7}-P, D_{21}=M_{5}{ }^{T} P A_{d}+$. $A_{c}{ }^{T} Q M_{7}, \quad D_{22}=M_{5}{ }^{T} P M_{5}+A_{c}{ }^{T} Q A_{c}+C_{C}{ }^{T} S C_{C}-Q$ $D_{31}=M_{6}{ }^{T} P A_{d}+M_{8}{ }^{T} Q M_{7}, D_{32}=M_{6}{ }^{T} P M_{5}+M_{8}{ }^{T} Q A_{c}$, $D_{33}=M_{6}{ }^{T} P M_{6}+M_{8}{ }^{T} Q M_{8}-S$.

By Schur complement, the above equation can be transformed to

$$
\left[\begin{array}{cccccc}
-P & * & * & * & * & * \\
0 & -Q & 0 & * & * & * \\
0 & 0 & -S & * & * & * \\
A_{d} & M_{5} & M_{6} & -P & * & * \\
M_{7} & A_{c} & M_{8} & 0 & -Q^{-1} & * \\
0 & C_{c} & 0 & 0 & 0 & -S^{-1}
\end{array}\right] .
$$

\section{[End of Proof]}

Theorem 5: For the plant in Figure 1, under dynamic output feedback controller, for $\gamma>0$, if there are symmetric positive definite matrices $\mathrm{P}, \mathrm{Q}, \mathrm{S}$ that

$\left[\begin{array}{cccccccc}-P & * & * & * & * & * & * & * \\ 0 & -Q & * & * & * & * & * & * \\ 0 & 0 & -S & * & * & * & * & * \\ 0 & 0 & 0 & -\gamma^{2} & * & * & * & * \\ A_{d} & M_{5} & M_{6} & W_{0} & -P^{-1} & * & * & * \\ M_{7} & A_{c} & M_{8} & W_{6} & 0 & -Q^{-1} & * & * \\ 0 & C_{c} & 0 & 0 & 0 & 0 & -S^{-1} & * \\ C_{21} & 0 & M_{4} & W_{5} & 0 & 0 & 0 & -I\end{array}\right]<0$,

then the plant in Fig. 1 realizes suboptimal dynamic output feedback $H_{\infty}$ control.

Proof: The external disturbance is considered in order to make the following equation exist $\|\mathrm{z}(\mathrm{k})\|_{2} \leq \gamma \quad \mid \mathrm{w}(\mathrm{k}) \|_{2}$.

Let $J_{Z}=\sum_{k=0}^{\infty}\left[z^{T}(k) z(k)-\gamma^{2} w^{T}(k) w(k)\right]$, choose positive definite matrices $\mathrm{P}, \mathrm{Q}, \mathrm{S}$, and construct a Lyapunov function $\mathrm{V}(\mathrm{k})=\mathrm{x}_{1}^{T}(k) P x_{1}(k)+x_{c}{ }^{T}(k)$ $\mathrm{Q} x_{c}(\mathrm{k})+. u^{T}(k-1) \mathrm{Su}(\mathrm{k}-1)$.

The dynamic output feedback close-loop system modeled in (2), if satisfies Theorem 4, the system is asymptotically stable in zero initial conditions for $\forall \mathrm{w}(\mathrm{k}) \in L_{2}[0, \infty)$. Then we have

$\sum_{k=0}^{\infty}\left[z^{T}(k) z(k)-\gamma^{2} w^{T}(k) w(k)\right]+\Delta V(k)<0$.

Let $W_{6}=B_{c}\left(H_{1}-C_{12} W_{2}\right), M_{4}=-C_{22} B_{2}, W_{5}=H_{2}-C_{22} W_{2}$, $\mathrm{x}=\left[\begin{array}{llll}\mathrm{x}_{1}^{T} & x_{c}{ }^{T} & u^{T} & w^{T}\end{array}\right]^{\mathrm{T}}$, we have $z^{T}(k) z(k)-$ $\left.\gamma^{2} w^{T}(k) w(k)\right]+\Delta V(k)=x^{T} \Omega \mathrm{x}$,

$\Omega=\left[\begin{array}{cccc}A_{11} & * & * & * \\ A_{21} & A_{22} & * & * \\ A_{31} & A_{32} & A_{33} & * \\ A_{41} & A_{42} & A_{43} & A_{44}\end{array}\right]$

$A_{11}=A_{d}{ }^{T} P A_{d}+M_{7}{ }^{T} Q M_{7}-P+C_{21}{ }^{T} C_{21}, A_{21}=M_{5}{ }^{T} P A_{d}$ $+A_{c}{ }^{T} Q M_{7}, A_{22}=M_{5}{ }^{T} P M_{5}+A_{c}{ }^{T} Q A_{c}+C_{C}{ }^{T} S C_{C}-Q, A_{31}=$ $M_{6}{ }^{T} P A_{d}+M_{8}{ }^{T} Q M_{7}+M_{4}{ }^{T} C_{21}, A_{32}=M_{6}{ }^{T} P M_{5}+M_{8}{ }^{T} Q A_{c}$, 


$$
\begin{aligned}
& A_{33}=M_{6}{ }^{T} P M_{6}+M_{8}{ }^{T} Q M_{8}-S+M_{4}{ }^{T} M_{4}, A_{41}=W_{0}{ }^{T} P A_{d}+ \\
& W_{5}{ }^{T} C_{21}+W_{6}{ }^{T} Q M_{7}, A_{42}=W_{0}{ }^{T} P M_{5}+W_{6}{ }^{T} Q A_{c}, A_{43}= \\
& W_{0}{ }^{T} P M_{6}+W_{5}{ }^{T} M_{4}+W_{6}{ }^{T} Q M_{8}, A_{44}=W_{5}{ }^{T} W_{5}-\gamma^{2}+W_{0}{ }^{T} P W_{0} \\
& +W_{6}{ }^{T} Q W_{6} .
\end{aligned}
$$

Now Equation (15) can be transformed to

$\left[\begin{array}{ccccccc}-P+C_{21}{ }^{T} C_{21} & 0 & * & * & * & * & * \\ 0 & -Q & * & * & * & * & * \\ M_{4}{ }^{\mathrm{T}} C_{21} & 0 & M_{4}^{\mathrm{T}} M_{4}-S & * & * & * & * \\ W_{5} \mathrm{~T} C_{21} & 0 & W_{5}^{\mathrm{T}} M_{4} & W_{5}^{\mathrm{T}} W_{5}-\gamma^{2} & * & * & * \\ A_{d} & M_{5} & M_{6} & W_{0} & -P^{-1} & * & * \\ M_{7} & A_{c} & M_{8} & W_{6} & 0 & -Q^{-1} & * \\ 0 & C_{c} & 0 & 0 & 0 & 0 & -S^{-1}\end{array}\right]<0$.

[End of Proof]

Theorem 6: For $\gamma>0$, if there exists a symmetric positive definite matrix $P=P^{T}>0$ which satisfies

$$
\left[\begin{array}{cccc}
(A+\Delta A)^{T} p+p((A+\Delta A))+I & p\left(A_{d}+\Delta A_{d}\right) & p(B+\Delta B) & C^{T} \\
\left(A_{d}+\Delta A_{d}\right)^{T} p & -I & 0 & 0 \\
(B+\Delta B)^{T} p & 0 & -\gamma^{2} I & 0 \\
C & 0 & 0 & -I
\end{array}\right]<0
$$

system (1) is robust stable.

Proof: From Schur complement lemma, (18) is equivalent to

$\left[\begin{array}{ccc}(A+\Delta A)^{T} p+p((A+\Delta A))+I & p\left(A_{d}+\Delta A_{d}\right) & p(B+\Delta B) \\ \left(A_{d}+\Delta A_{d}\right)^{T} p & -I & 0 \\ (B+\Delta B)^{T} p & 0 & -\gamma^{2} I\end{array}\right]+\left[\begin{array}{l}C^{T} \\ 0 \\ 0\end{array}\right]\left[\begin{array}{lll}C & 0 & 0\end{array}\right]<0$.

That is,

$\left[\begin{array}{ccc}(A+\Delta A)^{T} p+p((A+\Delta A))+I & p\left(A_{d}+\Delta A_{d}\right) & p(B+\Delta B) \\ \left(A_{d}+\Delta A_{d}\right)^{T} p & -I & 0 \\ (B+\Delta B)^{T} p & 0 & -\gamma^{2} I\end{array}\right]+\left[\begin{array}{l}C^{T} \\ 0 \\ 0\end{array}\right]\left[\begin{array}{lll}C & 0 & 0\end{array}\right]<0$.

(20)
$\left[(A+\Delta A)^{T} \mathrm{p}+p(A+\Delta A)+\right.$

$P(A d+\Delta A d)(A d+\Delta A d)^{T}$

$p+I+C^{T} C+\gamma^{-2} P(B+\Delta B)$

$\left.(B+\Delta B)^{T} p\right]<0$.

We consider Lyapunov functional

$$
\begin{aligned}
& J(x(t), t)=x^{T}(t) P x(t)+ \\
& \int_{0}^{t}\left(y^{T} y\right) d \varepsilon+\gamma^{-2} x^{T} P(B+\Delta B)^{T} P x d \varepsilon+ \\
& \int_{t-1}^{t} x(t+1)^{T}(t+1) d \varepsilon>0,
\end{aligned}
$$

differentiating with respect to $t$ at the both sides of $\mathrm{J}(\mathrm{x}(\mathrm{t}), \mathrm{t})$, we have

$$
\begin{aligned}
& \mathrm{J}(\mathrm{x}(\mathrm{t}), \mathrm{t})=\mathrm{x}^{\mathrm{T}}(\mathrm{t}) \operatorname{Px}(\mathrm{t})+x^{T}(t) \operatorname{Px} \&(\mathrm{t})+ \\
& x^{T}(t) C^{T} C x(t)+x^{T}(t) x(t)+ \\
& \gamma^{-2} x^{T} P(B+\Delta \mathrm{B})(B+\Delta \mathrm{B})^{T} P x(t)- \\
& x^{T}(t-l) x(t-l) .
\end{aligned}
$$

Then

$\mathrm{J}(\mathrm{x}(\mathrm{t}), \mathrm{t})=\mathrm{x}^{\mathrm{T}}(\mathrm{t})\left((\mathrm{A}+\Delta \mathrm{A})^{\mathrm{T}} \mathrm{P}+\mathrm{P}(\mathrm{A}+\Delta \mathrm{A})+\mathrm{I}+C^{T} C+\right.$ $\gamma^{-2} P(B+\Delta \mathrm{B})(B+\Delta \mathrm{B})^{T} P x(t)+$

$x^{T}(t-l)\left(A_{d}+\Delta A_{d}\right)^{\mathrm{T}} \mathrm{Px}(\mathrm{t})+\mathrm{x}^{\mathrm{T}}(\mathrm{t}) \mathrm{P}\left(A_{d}+\right.$

$\left.\Delta A_{d}\right)^{\mathrm{T}} \mathrm{x}(\mathrm{t}-\mathrm{l})-x^{T}(t-l) x(t-l)$,

further,

$\mathrm{J}(\mathrm{x}(\mathrm{t}), \mathrm{t})=\mathrm{x}^{\mathrm{T}}(\mathrm{t})\left((\mathrm{A}+\Delta \mathrm{A})^{\mathrm{T}} \mathrm{P}+\mathrm{P}(\mathrm{A}+\Delta \mathrm{A})+\right.$

$\mathrm{P}\left(A_{d}+\Delta A_{d}\right)\left(A_{d}+\Delta A_{d}\right)^{\mathrm{T}} \mathrm{P}+\mathrm{I}+C^{T} C+$

$\gamma^{-2} P(B+\Delta \mathrm{B})(B+\Delta \mathrm{B})^{T} P x(t)$

$\left(\left(A_{d}+\Delta A_{d}\right)^{T} \mathrm{Px}(\mathrm{t})-\mathrm{x}(\mathrm{t}-\mathrm{l})^{T^{*}}\left(A_{d}\right.\right.$

$\left.\left.+\Delta A_{d}\right)^{\mathrm{T}} \operatorname{Px}(\mathrm{t})-\mathrm{x}(\mathrm{t}-\mathrm{l})\right)$.

From (21), we have $J(x(t), t)<0$, so the plant (1) is robust stable.

[End of Proof] 


\section{Simulation Results}

1 Simulation of a Typical Singular Plant

We take state feedback case to illustrate the effectiveness of the proposed method. A typical singular plant model with input external disturbance is as follows:

$\left\{\begin{array}{c}{\left[\begin{array}{c}x_{1}(t) \\ 0 \\ x_{3}(t) \\ 0\end{array}\right]=.} \\ z\left(\begin{array}{cccc}0 & 1 & 0 & 0 \\ -1 & 0 & 0 & 1 \\ 1 & 0 & 0 & 0 \\ 0 & 1 & 1 & 1\end{array}\right]\left[\begin{array}{l}x_{1}(t) \\ x_{2}(t) \\ x_{3}(t) \\ x_{4}(t)\end{array}\right]+\left[\begin{array}{c}0 \\ 0 \\ 0 \\ -1\end{array}\right] \mathrm{u}(\mathrm{t}-\mathrm{l})+\left[\begin{array}{c}0 \\ 0 \\ 0 \\ 0.1\end{array}\right] \mathrm{w}(\mathrm{t}) . \\ z(t)=\left[\begin{array}{llll}1 & 1 & 1\end{array}\right] x(t)+0.1 w(t)\end{array}\right.$

The sampling period $T$ is $0.1 \mathrm{~s}$, the network-induced delay is $\tau_{\mathrm{k}}=0.01$. The plant model can be transformed as

$$
\left\{\begin{array}{c}
x_{1}(t)=\left[\begin{array}{cc}
-1 & -1 \\
1 & 0
\end{array}\right] x_{1}(t-l)+\left[\begin{array}{l}
1 \\
0
\end{array}\right] u(t)+\left[\begin{array}{c}
-0.1 \\
0
\end{array}\right] w(t) \\
0=x_{2}(t)+\left[\begin{array}{c}
-1 \\
0
\end{array}\right] u(t-l)+\left[\begin{array}{c}
0.1 \\
0
\end{array}\right] w(t) \\
z(t)=\left[\begin{array}{ll}
0 & 1
\end{array}\right] x_{1}(t)+\left[\begin{array}{ll}
1 & 1
\end{array}\right] x_{2}(t)+0.1 w(t)
\end{array} .\right.
$$

Its discrete model parameters are

$$
\begin{aligned}
A_{d} & =\left[\begin{array}{cc}
0.9 & -0.1 \\
0.1 & 1
\end{array}\right], B_{1}=\left[\begin{array}{l}
0.01 \\
0.01
\end{array}\right], \\
B_{2} & =\left[\begin{array}{c}
-1 \\
0
\end{array}\right], C_{21}=\left[\begin{array}{ll}
1 & 0
\end{array}\right], \\
C_{22} & =\left[\begin{array}{ll}
1 & 1
\end{array}\right], \text { and } H_{2}=0.1 .
\end{aligned}
$$

We find the solution of the plant through LMI toolbox using $u(t)=\left[\begin{array}{cccc}-5 & -4 & 0 & 0\end{array}\right] \mathrm{x}(\mathrm{t})$ for which the system is asymptotically stable. When initial state $\mathrm{x}(0)=(0,2,1,-$ 1) the system state response external trajectory since disturbance is as solid line shown in Fig. 2.

For $H_{\infty}$ control, we use Theorem 3. Therefore $\gamma=\sqrt{\beta}=35.92$ is obtained, and the $\gamma$-suboptimal state feedback $H_{\infty}$ control law is $\mathrm{u}(\mathrm{t})=[-0.290$ $\left.\begin{array}{lll}-0.034 & 0 & 0\end{array}\right] \mathrm{x}(\mathrm{t})$. Under the same conditions, the system state response trajectory is as dotted line shown in Figure 2.

By LMI tool-box, we present solutions for Theorem 4, the obtained corresponding solutions are

$$
\begin{aligned}
& \hat{S}^{*}=\left[\begin{array}{cc}
0.0951 & -0.0002 \\
-0.0001 & 0.1051
\end{array}\right], Y_{1}^{*}=1.0^{-6}\left[\begin{array}{ll}
-0.211 & 0.013
\end{array}\right], \\
& Y_{2}^{*}=Y_{3}^{*}=\left[\begin{array}{l}
0 \\
0
\end{array}\right], \beta^{*}=0.009 .
\end{aligned}
$$

Therefore, the minimum disturbance attenuation is $\gamma^{*}=\sqrt{\beta^{*}}=0.095$, , the $\gamma$-optimal state feedback $H_{\infty}$ control law is $\mathrm{u}(\mathrm{t})=1.0^{-5}\left[\begin{array}{llll}-0.22 & 0.01 & 0 & 0\end{array}\right] x(t)$.

After putting optimal $H_{\infty}$ into effect, the system state response trajectory is as dot dash line shown in Figure 2. Before and after optimization control, the system expectation output is presented as solid line and dotted line shown in Figure 3.

\section{Figure 2}

State response simulation

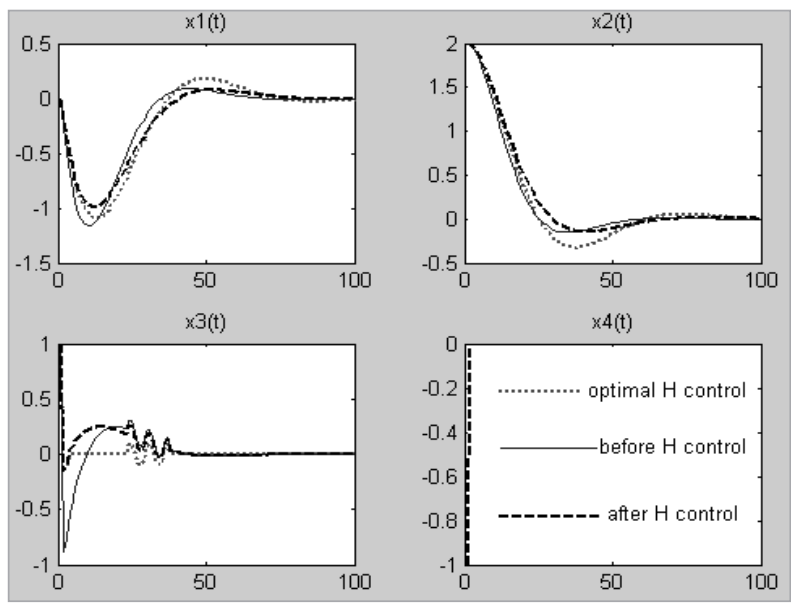

Figure 3

Expectation output simulation

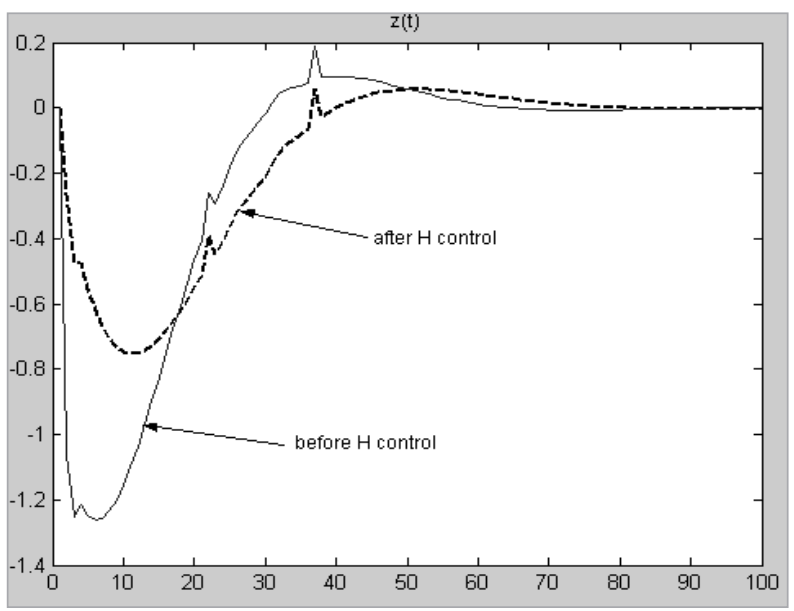

Further,

$$
\begin{aligned}
& \hat{s}=\left[\begin{array}{cc}
0.097 & -0.015 \\
-0.015 & 0.094
\end{array}\right], Y_{1}=\left[\begin{array}{ll}
-0.028 & 0.001
\end{array}\right], \\
& Y_{2}=Y_{3}=\left[\begin{array}{l}
0 \\
0
\end{array}\right], \beta=1288.6,
\end{aligned}
$$


and the system simulation shows that after implementation $H_{\infty}$ control and $H_{\infty}$ optimization control, $\gamma$ can decrease to 0.06 from the primary 35.9 , and the anti-interference performance of the system is enhanced markedly. As a result, the stability performance of system has been improved.

\section{Simulation of a Torpedo}

The longitudinal motion of the dynamic equation of a torpedo at the speed $v=25.7 \mathrm{~m} / \mathrm{s}$ can be described by the following state equation:

$$
\left\{\begin{aligned}
x \&= & {\left[\begin{array}{cc}
-1.4 & 0.22+0.17 \delta_{2} \\
10+0.25 \delta_{1} & -5.4
\end{array}\right] x+} \\
& {\left[\begin{array}{cc}
-1.3 & 0.22-0.25 \eta_{2} \\
10-0.25 \eta_{1} & -5
\end{array}\right] x(t-l)+} \\
& {\left[\begin{array}{cc}
-0.28 & -0.03 \delta_{2} \\
-0.03 \delta_{1} & -4.13
\end{array}\right] \delta_{e}, } \\
y(t)= & {\left[\begin{array}{ll}
1 & 0 \\
0 & 1
\end{array}\right] x }
\end{aligned}\right.
$$

where $x_{1}$ is the attack angle of the torpedo, $x_{2}$ is the angular velocity of the torpedo, $\delta_{e}$ is the rudder angle of the torpedo, and $\left|\delta_{i}\right| \leq 1,\left|\eta_{i}\right| \leq 1, i=1,2$,

the simulation result is shown in Figure 4.

Figure 4

Torpedo system simulation

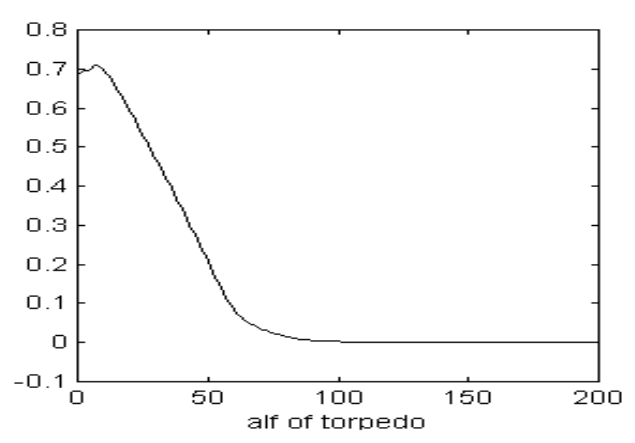

\section{References}

1. Gahinet, P. Explicit Controller Formulas for LMI-based Hœ Synthesis. Automatica, 1996, 32(7), 1201-1212. https://doi.org/10.1016/0005-1098(96)00033-7

2. Grigoriadis, K. M., Watson, J. T., Jr. Reduced-Order $\mathrm{H} \infty$ and L2- L $\infty$ Filtering via Linear Matrix Inequalities. IEEE Transactions on Aerospace and Electronic Systems, 1997, 33(4), 1326-1338. https://doi. org/10.1109/7.625133

\section{Conclusions}

In this paper, the $H_{\infty}$ optimal control problems for a class of SNCS have been addressed with both state feedback case and dynamic output feedback case. The network communications characteristics in the paper are: network-induced delay, input disturbance of limited energy, clock-driven sensors, event-driven controller and actuators. The characteristics for singular system in the paper are: impulse behavior, structural instability, and something like that. When network communication time-delay is less than or equal to a sampling in both cases state the feedback and dynamic output feedback are correct. This paper presents respectively the existence condition of the $H_{\infty}$ control law, $H_{\infty}$ optimal control method, and solution of $H_{\infty}$ control law. The simulation results show that the analytical method and the results are valid and feasible.

\section{Acknowledgement}

We would like to thank the anonymous reviewers for their valuable comments. This work is supported by his research was financially supported by the Open Research Fund from Shandong provincial Key Laboratory of Computer Network, Grant No.: SDKLCN-2017-02. This job is also supported by China Postdoctoral Science Foundation (No. 2013M542370), the Specialized Research Fund for the Doctoral Program of Higher Education of China (Grant No. 20136118120010), Natural Science Foundation of China (No. 61272509), Beijing Natural Science Foundation (No. 4132049) and supported by Scientific Research Program Funded by Shaanxi Provincial Education Department (Program No. 2013JK1139).

3. Ji, X. F., Su, H. Y., Chu, J. An LMI Approach to Robust Stability of Uncertain Discrete Singular Time-Delay Systems. Asian Journal of Control, 2006, 8(1), 56-62. https://doi.org/10.1111/j.1934-6093.2006.tb00252.x

4. Kim, J. H. Delay-Dependent Robust $\mathrm{H} \infty$ Filtering for Uncertain Discrete-Time Singular Systems with Interval Time-Varying Delay. Automatica, 2010, 46(3), 591597. https://doi.org/10.1016/j.automatica.2010.01.011 
5. Lan, Q.- X., Liu, Y. X., Niu, H. W., Liang, J. Robust Reliable Guaranteed Cost Control for Uncertain Singular Systems with Time-Delay. Journal of Systems Engineering and Electronics, 2010, 21(1), 110-117. https:// doi.org/10.3969/j.issn.1004-4132.2010.01.018

6. Ma, S. P., Boukas, E. K., Chinniah, Y. Stability and Stabilization of Discrete-Time Singular Markov Jump Systems with Time-Varying Delay. International Journal of Robust and Nonlinear Control, 2010, 20(5), 531-543.

7. Qiang, Y., Zhang, J. A Bijection Between Lattice-Valued Filters and Lattice-Valued Congruences in Residuated Lattices. Mathematical Problems in Engineering, 2013, 36(8), 4218-4229.

8. Song, Y., Wang, J., Shi, Y., Li, C. Packet-Loss-Dependent Stabilization of NCSs with Network-Induced Delay and Packet Dropout. Journal of Systems Engineering and Electronics, 2012, 23(3), 408-413. https://doi. org/10.1109/JSEE.2012.00051

9. Srivastava, H. M., Zhang, Y., Wang, L., Shen, P., Zhang, J. A Local Fractional Integral Inequality on Fractal Space Analogous to Anderson's Inequality. Abstract and Applied Analysis, 2014, 46(8), 5218-5229. https://doi. org/10.1155/2014/797561

10. Sun, X., Zhang, Q., Yang, C., Su, Z. Stability Analysis and Stabilization for Discrete-Time Singular Delay Systems. Journal of Systems Engineering and Electronics, 2011, 22(3), 482-487. https://doi.org/10.3969/j. issn.1004-4132.2011.03.017

11. Wei, W., Fan, X., Song, H., Wang, H. Video Tamper Detection Based on Multi-Scale Mutual Information. Multimedia Tools and Applications, 2017, 1-18. https:// doi.org/10.1007/s11042-017-5083-1

12. Wei, W., Fan, X., Song, H., Fan, X., Yang, J. Imperfect Information Dynamic Stackelberg Game Based Resource Allocation Using Hidden Markov for Cloud Computing. IEEE Transactions on Services Computing, 2016, PP(99), 1-13. https://doi.org/10.1109/ TSC.2016.2528246

13. Wei, W., Qi, Y. Information Potential Fields Navigation in Wireless Ad-Hoc Sensor Networks. Sensors, 2011, 11(5), 4794-4807. https://doi.org/10.3390/s110504794

14. Wei, W., Song, H., Li, W., Shen, P., Vasilakos, A. Gradient-Driven Parking Navigation Using a Continuous Information Potential Field Based on Wireless Sensor Network. Information Sciences, 2017, 408(C), 100-114. https://doi.org/10.1016/j.ins.2017.04.042
15. Wei, W., Song, H., Wang, H., Fan, X. Research and Simulation of Queue Management Algorithms in Ad Hoc Network under DDoS Attack. IEEE Access, 2017, 5, 2781027817. https://doi.org/10.1109/ACCESS.2017.2681684

16. Wei, W., Sun, Z., Song, H., Wang, H., Fan, X. Energy Balance-Based Steerable Arguments Coverage Method in WSNs. IEEE Access, 2017, 5, 27810-27817. https://doi. org/10.1109/ACCESS.2017.2682845

17. Xu, Q., Wang, L., Hei, X.-H., Shen, P., Shi, W., Shan, L. GI/Geom/1 Queue Based on Communication Model for Mesh Networks. International Journal of Communication Systems, 2014, 27 (11), 3013-29.

18. Xu, S. Y., Van Dooren, P., Stefan, R., Lam, J. Robust Stability and Stabilization for Singular Systems with State Delay and Parameter Uncertainty. IEEE Transactions on Automatic Control, 2002, 47(7), 1122-1128. https:// doi.org/10.1109/TAC.2002.800651

19. Yang, X.-L., Shen, P.-Y., Zhou, B. Holes Detection in Anisotropic Sensornets: Topological Methods. International Journal of Distributed Sensor Networks, 2012, ID 135054. https://doi.org/10.1155/2012/135054

20. Yang, F. W., Wang, Z. D., Hung, Y. S., Gani, M. Hœ Control for Networked Systems with Random Communication Delays. IEEE Transactions on Automatic Control, 2006, 51(3), 511-518. https://doi.org/10.1109/ TAC.2005.864207

21. Yang, X.-L., Zhou, B., Feng, J., Shen, P.-Y. Combined Energy Minimization for Image Reconstruction from Few Views. Mathematical Problems in Engineering, 2012, 1-15. https://doi.org/10.1155/2012/154630

22. Yue, D., Han, Q. L., Lam, J. Network-Based Robust $\mathrm{H} \infty$ Control of Systems with Uncertainty. Automatica, 2005, 41(6), 999-1007. https://doi.org/10.1016/j.automatica.2004.12.011

23. Zhang, W., Yu, L. Output Feedback Stabilization of Networked Control Systems with Packet Dropouts. IEEE Transactions on Automatic Control, 2007, 52(9), 17051709. https://doi.org/10.1109/TAC.2007.904284

24. Zhang, W., Branicky, M. S., Phillips, S. M. Stability of Networked Control Systems. IEEE Control Systems Magazine, 2001, 21(1), 84-99. https://doi. org/10.1109/37.898794

25. Zhivoglyadov, P. V., Middleton, R. H. Networked Control Design for Linear Systems. Automatica, 2003, 39(4), 743-750. https://doi.org/10.1016/S00051098(02)00306-0 